\title{
Predicting Drivers' Direction Sign Reading Reaction Time Using an Integrated Cognitive Architecture
}

\author{
Chao Deng ${ }^{1,2,3}$, Shi $\mathrm{Cao}^{4}$, Chaozhong Wu ${ }^{1,2,3,}$, Nengchao Lyu ${ }^{1,2,3}$ \\ ${ }^{1}$ Intelligent Transportation Systems Research Centre, Wuhan University of Technology, Wuhan, Hubei, China \\ ${ }^{2}$ Engineering Research Centre for Transportation Safety, Wuhan University of Technology, Wuhan, Hubei, \\ China \\ ${ }^{3}$ National Engineering Research Centre for Water Transport Safety, Wuhan University of Technology, Wuhan, \\ Hubei, China \\ ${ }^{4}$ Department of Systems Design Engineering, University of Waterloo, Waterloo, Ontario N2L 3G1, Canada \\ *wucz@whut.edu.cn
}

\begin{abstract}
Objective. Drivers' reaction time of reading signs on expressways is a fundamental component of sight distance design requirements, and reaction time is affected by many factors such as information volume and concurrent tasks. In this study, we built cognitive simulation models to predict drivers' direction sign reading reaction time. Method. Models were built using the Queueing Network-Adaptive Control of Thought Rational (QN-ACTR) cognitive architecture. Drivers' task-specific knowledge and skills were programmed as production rules. Two assumptions about drivers' strategies were proposed and tested. The models were connected to a driving simulator program to produce prediction of reaction time. Model results were compared to human results in both sign reading single-task and reading while driving dual-task conditions. The models were built using existing modelling methods without adjusting any parameter to fit the human data. Results. The models' prediction was similar to the human data and could capture the different reaction time in different task conditions with different numbers of road names on the direction signs. Root mean square error (RMSE) was $0.3 \mathrm{~s}$, and mean absolute percentage error (MAPE) was $12 \%$. Conclusion. The results demonstrated the models' predictive power. The models provide a useful tool for the prediction of driver performance and the evaluation of direction sign design. Key words: Driving safety, Direction sign design, Reaction time, QN-ACTR, Concurrent tasks
\end{abstract}

\section{Introduction}

Direction signs play an important role in driving safety and accident prevention [1]. Direction signs are traffic signs that "show route designations, destinations, directions, and other geographical information [2 p. 28]." Proper use of direction signs can improve traffic flow and reduce accidents. In general, direction signs should be designed following standards and visibility principles to avoid confusion and minimize drivers' mental effort in interpreting direction signs. From a cognitive perspective, drivers' cognitive resources are limited. Searching and identifying information on direction signs require time, and reaction time usually increases with the amount of information on a direction sign [1]. Therefore, the amount of information (i.e., information volume) on a direction sign should be designed properly, considering drivers' cognitive limitations. For example, a lot of direction signs are used on highways and expressways. If they contain too much information, drivers may not have enough time to process the information when driving at a high speed; if they contain too little information, it may not be sufficient to provide guidance in complex route conditions. The reaction time of reading direction signs on expressways is a fundamental component of sight distance design requirements, and proper information design of direction signs is crucial to driving safety [3].

To determine the proper amount of information on direction signs, research is needed to study the relationship between information amount and direction sign reading reaction time and build models that can predict reaction time in different conditions. Direction sign reading reaction time is defined as the duration from the onset of a direction sign, i.e., when it is visible to a driver, to the moment when the driver finishes processing necessary information on the sign, often indicated by pressing a button as the response. The reaction time has been measured in both static and dynamic tests. In static tests, participants read direction signs shown on a display without concurrent driving tasks. In dynamic tests, participants read direction signs while driving, which is often conducted using driving simulators. In each trial of the test, multiple items such as road names are presented on a sign, and participants are usually asked to press keys as their responses indicating the location of the target item. It is commonly accepted that sign reading reaction time is longer when a larger amount of information presents on the sign. For example, Du et al. [4] measured direction sign reading reaction time in a static test and found that reaction time significantly increased as the total number of road names on a sign increased. Similarly, in a dynamic test using a driving simulator, Lyu et al. [5] found that reaction time of reading highway direction signs significantly increased as the number of road names increased, whereas driving experience and gender had no significant effect on the reaction time. Direction sign reading reaction time is usually longer in dynamic tasks than in static tasks, because while driving, drivers need to switch the focus of visual attention between different areas of interest such as signs, road users, and roadway [6].

A widely used method to quantify information is information entropy [7]. In choice reaction tasks, each trial has one item as the target item among all displayed items. 
With the assumption that each item has an equal probability to be the target, the information volume in choice reaction tasks is $\log _{2} N$, where $N$ is the number of items in a trial. This information entropy method has been adopted to quantify information volume of direction signs $[1,8]$. Regarding the relationship between stimulus information volume and reaction time, Hick-Hyman law states that choice reaction time increases with information volume following a linear relationship [9, 10]. However, information entropy models have their limitations for the prediction of direction sign reading reaction time. The entropy models are regression models that consider only one factor, which is the amount of information. Other factors such as road condition, traffic condition, driver fatigue, and driver attention also affect sign reading reaction time, but the regression models cannot account for these factors. One way to address this issue is using simulation models based on cognitive architectures.

A cognitive architecture is both a unified theory of cognition and a computerized simulation program. Theoretically, a cognitive architecture combines cognitive psychology theories about different components of cognition, so it can explain the effects of different cognitive factors that impact human behavior. Practically, a cognitive architecture can serve as an engineering tool for the prediction and simulation of human performance in different human-machine interaction tasks. Examples of cognitive architectures include Adaptive Control of Thought-Rational (ACT-R) [11], Soar [12], and Queuing Network (QN) cognitive architectures [13, 14]. In particular, Queueing Network-Adaptive Control of Thought Rational (QN-ACTR) is a recent cognitive architecture that integrates $\mathrm{QN}$ and ACT-R architectures, combining the advantages of modeling multitasking performance in Queuing Network methods and the advantages of modeling complex cognitive activities in ACT-R [13].

Because this study focuses on QN-ACTR, we provide a brief introduction to the fundamental mechanisms of ACT-R and the connection between QN-ACTR and ACT-R. ACT-R's major components include the architecture of mental modules, model parameters, and task specific knowledge. The architecture represents the structure of human cognition as multiple connected mental modules, such as long-term memory and visual perception. Each module has algorithms to simulate the mechanism of information processing based on cognitive psychology literature. Model parameters represent the capacity and limitation of human mental processing. To model a specific task, the architecture is supplied with task specific knowledge including both production rules (procedural knowledge) and chunks (declarative knowledge). A production rule is a condition-action (if-then) pair. It can only be executed when its condition part matches the model's current mental state, which consists of the states of different mental modules. ACT-R has algorithms for rule selection and learning based on rule utility, which represents the relative desirability to execute the rule. Every production rule in ACT-R has a firing cycle of $50 \mathrm{~ms}$ by default. Multiple rules matched in the same cycle will compete because only one rule is allowed to execute within a firing cycle. ACT-R also has algorithms for calculating chunk activation level, which determines chunk retrieval time and retrieval probability. Mental processing is simulated as the cognitive architecture utilizing production rule and chunk knowledge to reach a pre-defined goal.

Along another line of reach, QN was used to model human performance time and error [15]. While ACT-R models traditionally focused on single-tasks, an advantage of QN is to simulate multitasking performance using queueing schemes as Liu stated in 1997 [16]. In a following QN work in 2002, "If more than one goal may be executed (i.e., a multiple task scenario), ..., three schemes have been included: one, randomly select between the various goals; two, choose the goal which has waited the longest since its last execution; or three, select the goal with the highest priority value in the goal list array" [17 p. 138]. This queueing mechanism was later adopted in 2008 as threaded cognition [18] implemented in ACT-R, whose core assumption is "When multiple threads contend for the procedural resource, the least recently processed thread is allowed to proceed" [18 p. 111], so threaded cognition can be considered as one kind of queueing mechanism.

In QN-ACTR, queueing mechanisms were further integrated with ACT-R, by introducing queues to ACT-R modules [19] and a filtering discipline to production rule scheduling [20], both of which have been shown necessary for modeling multitasking performance. The multitask scheduling mechanism in QN-ACTR considers both the need to share limited mental resources across multiple concurrent tasks and the need to maintain the continuation of each task. To achieve resource sharing across multiple tasks, a natural queueing mechanism states that the task with the longest waiting time receives priority. To maintain necessary continuation within each task, a filtering discipline has been established to confine procedural module processing resources within the same task for the duration needed. This filtering discipline is unique in QN-ACTR and neither covered in previous QN work nor threaded cognition work. The logic is that some production rules require temporary information stored in peripheral buffers, for example, a chunk of declarative knowledge just retrieved from the long-term memory or a visual text just encoded in the visual module. If one task is interrupted by another task, such temporary information can be cleared from the buffers, and multitask performance will break down. The filtering discipline enforces restriction that allows one task to exclusively hold resources and complete necessary processing before releasing the resources back to other tasks. The filtering discipline states that a production rule that requires follow-up processing can prevent production rules from other tasks being selected until another production rule from the same task is selected for execution. A production rule is categorized as requiring follow-up if it has any action including declarative retrieval request, imaginal creation request, aural-location request, aural encoding request, visual-location request, and visual encoding request.

We chose to use QN-ACTR in the current study, because previous studies along this line of research have prepared the foundation for visual search models and driving models. A driving model was proposed and examined by Salvucci in 2006 . The model combined visual search modeling methods in ACT-R and a control function that determined drivers' incremental control movements in each control cycle [21]. The model was able to simulate driving behaviors including lane keeping, lane changing, and car following. Subsequently, this modeling method has 
been adopted in a series of studies that modeled the effect of driving experience on collision avoidance braking [22], the development of vehicle lateral control skills [23], as well as driving with concurrent tasks such as driving with a memory rehearsal task [24] and driving with a speech comprehension task [25]. However, previous studies have not modeled driving with a concurrent direction sign reading task. According to multiple resource theory [26], driving (specifically, vehicle control) is a task that mainly relies on visual attention, and therefore it should has more interference with concurrent direction sign reading than with concurrent speech comprehension, because both driving and direction sign reading require the same visual attention resources.

In the current study, we built models using the QN-ACTR cognitive architecture to predict direction sign reading reaction time in both static and dynamic tests. QN-ACTR was selected because previous studies along this line of research have provided a working model of driving behavior as well as a simulation platform that connects QN-ACTR with a driving simulator [27]. We focused on direction signs on expressways, because direction signs often contain a relatively large amount of information (i.e., many road names), and drivers need to read them quickly and accurately. To validate the models, we compared model reaction time with human reaction time obtained in the same tasks. The human data were taken from previous empirical studies [5]. In the method section, we describe the human empirical studies and the modeling details. The comparison between human results and model results is presented in the result section.

\section{Method}

To simulate and predict direction sign reading reaction time, we first built a model for the sign reading single task. Because QN-ACTR is a production rule system, we defined drivers' task-specific knowledge and skills using a series of production rules. To predict reaction time in the dual-task condition, we built the dual-task model by combining production rules for the sign reading task and production rules for the driving task, directly using previous driving models. QN-ACTR's multitask scheduling mechanism was used to manage the demands from both reading and driving tasks when they competed for limited attention and cognitive resources. Both static and dynamic tests, same as the ones used in the human study, were programmed in the simulation platform and presented to the models. Simulation was performed to collected model results, which were then compared with corresponding human results available from a previous study [5]. All parameters in the cognitive architecture were using their default values without adjusting any parameter to fit the human data.

\subsection{Human Study}

The human data were from two empirical experiments that have been reported previously [5]. Experiment 1 was a static test in which participants read direction signs shown on a computer display (single-task). Experiment 2 was a dynamic test in which participants read direction signs while driving on an expressway in a simulator (dual-task). The two experiments were conducted separately and used different direction sign materials. In this section, we provide a brief review of the two human experiments.

Forty-four drivers participated in both experiments. Their age ranged from 22 to 54 years ( $\mathrm{SD}=10$ years) with an average of 36 years. Their average driving mileage was about $11,000 \mathrm{~km}$ per year.

The static test in Experiment 1 was presented on a computer display, and participants were asked to find a target road name shown in each trial. A direction sign was presented in each trial, and multiple road names on the sign were evenly grouped into three direction groups, including leftward, forward, and rightward. Participants were asked to determine the direction of the target road as soon as possible and press the corresponding key on a keyboard as the response. Direction signs were designed following Chinese national standards with blue background and white text (Fig. 1) [28]. Multiple road names were evenly grouped into three direction groups, including leftward, forward, and rightward. The height of each Chinese character was $1.9 \mathrm{~cm}$ (visual angle 1.56 degree) on the display that was placed approximately $70 \mathrm{~cm}$ in front of the participants' eyes. The signs were categorized into three information levels depending on the total number of road names shown on each sign. The levels included Level 1 (three road names), Level 2 (six road names), and Level 3 (nine road names). Reaction time was recorded as the duration from the onset of a sign to the moment of response. Incorrect responses were excluded from the calculation of reaction time.

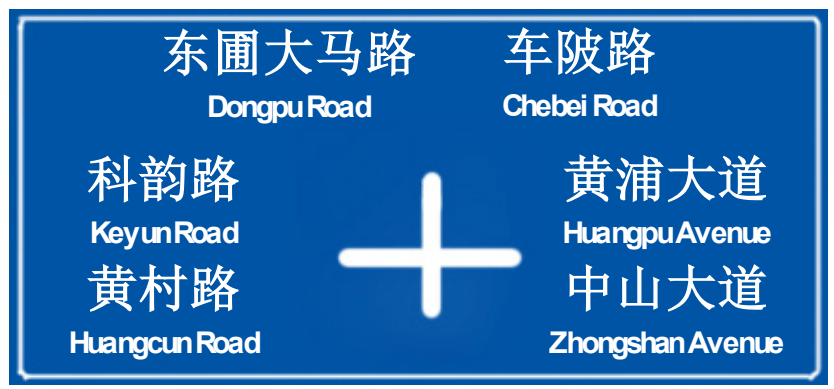

Fig. 1. Example of direction signs in Experiment 1

The dynamic test in Experiment 2 was conducted using a fixed-base driving simulator. Participants drove a car in a simulated expressway scenario without other traffic while reading direction signs at the same time. Direction signs were designed following Chinese national standards for expressway with designed speed over $80 \mathrm{~km} / \mathrm{h}$ [28], using green background and white text (Fig. 2). Direction signs were presented on a computer display separated from the driving simulator. The height of each Chinese character was $3.7 \mathrm{~cm}$ (visual angle 3.03 degree) on the display that was placed approximately $70 \mathrm{~cm}$ in front of the participants' eyes. The total number of road names on the signs had three levels including Level 1' (six road names), Level 2' (twelve road names), and Level 3' (eighteen road names). The road names on each sign were divided into two direction groups, i.e., forward and rightward. In Level 1' and 2', road names on each sign were evenly divided into the two directions. In Level 3', the forward group had 12 road names, and the rightward group had 6 road names. Participants were asked to maintain steady control of the car while responding to 
direction signs by pressing two buttons on the steering wheel to indicate the direction of the target road name.

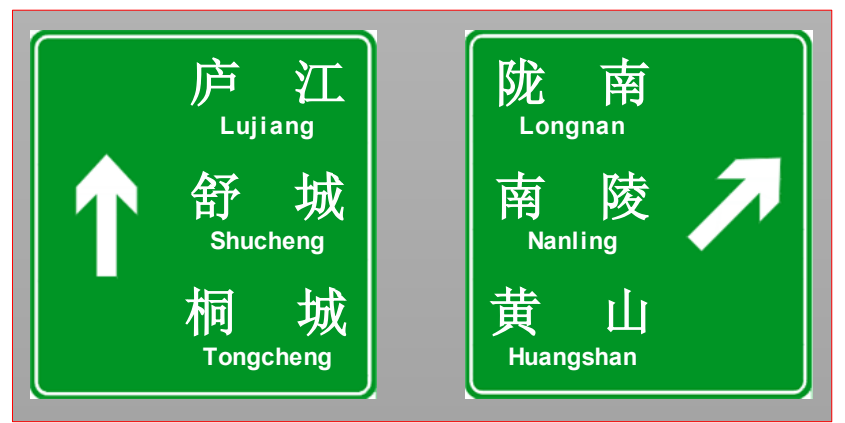

Fig. 2. Example of direction signs in Experiment 2

\subsection{Modelling and Simulation}

2.2.1 Direction sign Reading Model: A direction sign reading model was built for the direction sign reading single task. The production rules were defined following previous models with similar visual search and reading task components [29]. Two assumptions were made about drivers' visual search and response strategies.

Assumption 1: Participants used ordered serial visual search rather than random search and therefore avoided attending to the same road name more than once. This assumption is reasonable because people are familiar with reading words following a certain order. By scanning through all the road names on the sign in an order, drivers can avoid scanning the same location more than once. This assumption was implemented using a visual module mechanism built in the cognitive architecture that allows the search of unattended visual location to follow a specific order. In particular, a left-first order was used. When the model searched for an unattended visual location, it would select the one closest to the left. If multiple road names had the same horizontal location, a top-first order was then used to select the one from them that was closest to the top. The model could remember the visual locations that had been scanned, so it would not re-visit them in future visual search.

Assumption 2: Participants used a heuristic strategy (shortcut) that could determine the target road name was within the last direction group after scanning through the other direction groups without seeing the target road name. In the human experiments, participants were told the fact that the target road name was included in every trial. For example, in a trial with nine road names grouped in three directions, leftward, forward, and rightward each three road

Table 1 Procedures and production rules for guide sign reading single task

\begin{tabular}{ll}
\hline Production rule name & Task procedure (description of the source code) \\
\hline find-unattended-name* & IF the goal state is start, \\
& $\begin{array}{l}\text { THEN find an unattended visual-location in the visual field that is closest to the left, and change the goal } \\
\text { state to find-location. }\end{array}$
\end{tabular}

attend-name* IF the goal state is find-location, a visual-location has been found, and the visual module is currently free,

THEN move visual attention to the visual-location to encode its information, change the goal state to attend, and store the coordinate of the visual-location in the goal buffer.

respond-right (shortcut) IF the goal state is find-location, a visual-location has been found with a coordinate that belongs to the rightward direction group (this means that the road names in the leftward and upward groups all have been attended, but still the target is not found), and the manual module is free,

THEN press the key representing the rightward answer (e.g., key j), and the trial is done.

encode-name* IF the goal state is attend, visual module has encoded a road name, and imaginal module is free,

THEN create a mental representation of the road name in the imaginal buffer, and change the goal state to respond.

respond-left IF the goal state is respond, the road name mental representation is the same as the target road name, the manual module is free, and the coordinate of the road name is within the leftward direction group,

THEN press the key representing the leftward answer (e.g., key f), and the trial is done.

respond-up

IF the goal state is respond, the road name mental representation is the same as the target road name, the manual module is free, and the coordinate of the road name is within the upward direction group,

THEN press the key representing the upward answer (e.g., key y), and the trial is done.

read-again

IF the goal state is respond, and the road name mental representation is not the same as the target road name,

THEN, change the goal state back to start.

\footnotetext{
*: production rules that require follow-up processing (explained in the dual-task model section).

Note: when these production rules were adapted to the dual-task model, respond-left had no effect in the dual-task condition, because there was no leftward direction in Experiment 2. In Level 3' of Experiment 2, the visual search order (in find-unattended-name) was changed to right-first (i.e., first attending location closest to the right), because there were fewer road names in the rightward group (six names) than the forward group (twelve names).
} 
Table 2 Procedures and production rules for the driving task

\begin{tabular}{ll}
\hline Production rules & \multicolumn{1}{c}{ Task procedure (description of the source code) } \\
\hline drive-control-attend-near* & At the start of each steering control cycle, look for visual-location of near-point. \\
drive-control-process-near-attend-far* & IF a near-point is focused in visual-location buffer, \\
& THEN update near-point information in the goal buffer, and look for visual-location of \\
& far-point. \\
drive-control-process-far & IF a far-point is focused in visual-location buffer, \\
& THEN update far-point information in the goal buffer, and send motor commands to \\
steer the wheel and control the pedals.
\end{tabular}

*: production rules that require follow-up processing.

names, if participants did not find the target among the six road names of the leftward and forward groups, the target must be within the rightward group. Participants were asked to response as fast as possible and were given time to practice the trials. Using this shortcut strategy could lead to faster responses, especially when there were many road names.

Implementing the two assumptions, a model was built in QN-ACTR (source codes are available at https://github.com/HOMlab/QN-ACTR-Release) to simulate human performance in the direction sign reading single task. The production rules and their descriptions are shown in Table 1. Each production rule represents a step in the task procedure. The definition of these production rules follows the modeling principles used in previous cognitive models. The goal state initiated from start at the beginning of each trial. All parameters were using their default values.

2.2.2 Driving Model: A direction sign reading model was built for the direction sign reading single task. The production rules were defined following previous models with similar visual search and reading task components [29]. Two assumptions were made about drivers' visual search and response strategies.

To simulate driving performance, a model used in previous work was adopted [21, 23, 25]. This model assumes that drivers adjust steering wheel angle and accelerator and brake pedal positions according to the perceived information such as road heading direction and speed. Two visual reference points to extract such information are defined as near-point and far-point in front of the driver's vehicle. The information from the two points represents the road heading direction and is used as input to a control function, which determines the adjustment of steering wheel angle [30]. Three production rules are defined to complete each control cycle. By default, the execution of each production rule takes $50 \mathrm{~ms}$, so the duration of each control cycle is typically $150 \mathrm{~ms}$. The descriptions of the production rules are shown in Table 2. All parameters were using their default values.

\subsubsection{Dual-task Model of Sign Reading While Driving:}

The two single-task models introduced previously were combined to form the dual-task model for the simulation of direction sign reading while driving. The general methods and principles of combining single-task models to form a multitask model have been demonstrated in previous work of the QN-ACTR literature $[31,13,25]$ and mentioned in the introduction section. Following the filtering discipline, three production rules from the sign reading model and two production rules from the driving model require follow-up processing. These production rules are marked in Table $\mathbf{1}$ and 2.

2.2.4 Simulation: The sign reading tests for the models were programmed to be the same as corresponding tests in the human experiments. To simulate sign reading while driving, the mental model built in QN-ACTR was connected with a driving simulation program TORCS (http://torcs.sourceforge.net) via User Datagram Protocol (UDP), showing the same expressway scenario as in human Experiment 2. In each experimental condition, the model simulation was repeated for 600 trials of sign reading, reaching a criterion that the width of $95 \%$ confidence interval of reaction time was within 200 ms. Average reaction time from each experimental condition was calculated and compared with corresponding human results. To examine Assumption 1 and 2, alternative models without Assumption 1 or Assumption 2 were also tested.

\section{Results}

Regarding the human results, direction sign reading reaction time was summarized below for each condition in Experiment 1 and 2. In Experiment 1 (sign reading single task), mean reaction time was $1.2 \mathrm{~s}$ for Level $1(\mathrm{SD}=0.1 \mathrm{~s})$, $1.5 \mathrm{~s}$ for Level $2(\mathrm{SD}=0.1 \mathrm{~s})$, and $2.4 \mathrm{~s}$ for Level $3(\mathrm{SD}=$ 0.2 s) respectively. In Experiment 2 (sign reading while driving dual task), mean reaction time was $2.4 \mathrm{~s}$ for Level 1 ' $(\mathrm{SD}=0.2 \mathrm{~s}), 3.0 \mathrm{~s}$ for Level 2' $(\mathrm{SD}=0.2 \mathrm{~s})$, and $3.7 \mathrm{~s}$ for Level 3' $(\mathrm{SD}=0.3 \mathrm{~s})$ respectively.

Regarding the model results, for the sign reading

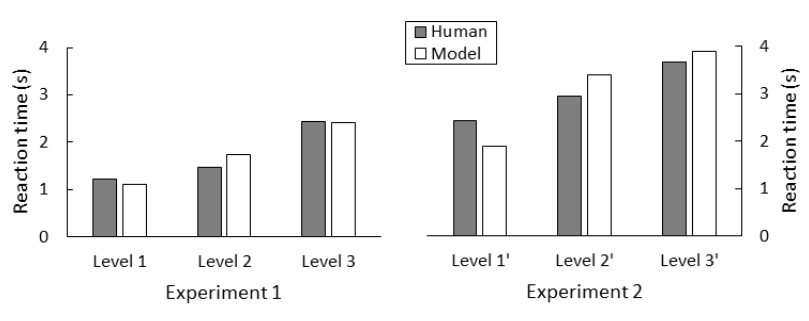

Fig. 3. Sign reading reaction time from different conditions for both human and model 
single task, predicted reaction time was $1.1 \mathrm{~s}$ for Level $1,1.7$ $\mathrm{s}$ for Level 2, and $2.4 \mathrm{~s}$ for Level 3 respectively. For the sign reading while driving dual task, predicted reaction time was $1.9 \mathrm{~s}$ for Level 1', $3.4 \mathrm{~s}$ for Level 2', and $3.9 \mathrm{~s}$ for Level 3' respectively. For comparison, the human and model results were plotted in Fig. 3. Regarding overall model fitness, root mean square error (RMSE) was $0.3 \mathrm{~s}$, and mean absolute percentage error (MAPE) was $12 \%$.

In order to examine the necessity of Assumption 1, we tested an alternative model with Assumption 1 removed. As shown in Fig. 4, the reaction time results from this alternative model were much longer than the human results in the Level 3' condition of the dual task, when the driving task caused some delay in reaction and the number of road names was the largest (eighteen road names). Without Assumption 1, the model could forget the locations previously attended and attend them again, causing longer reaction time. The MAPE increased to $32 \%$, and RMSE increased to $1.9 \mathrm{~s}$. This result showed that Assumption 1 is necessary to accurately reflect human performance.
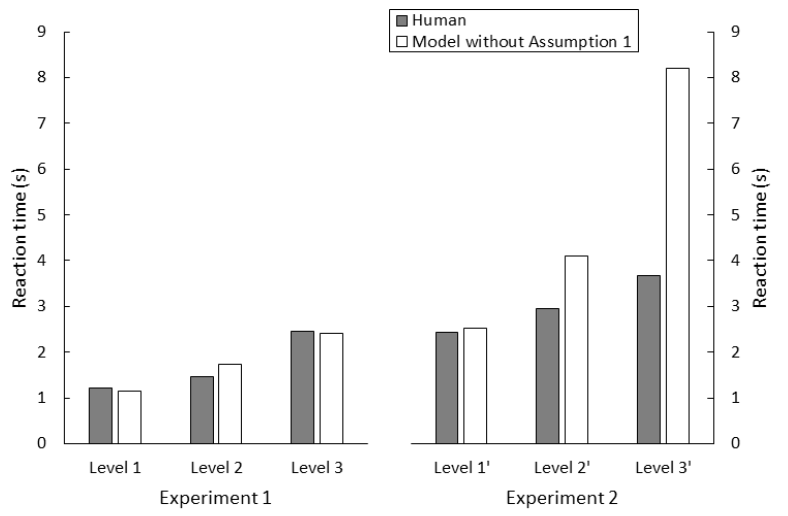

\section{Fig. 4. Sign reading reaction time from different}

Similarly, we tested another alternative model with Assumption 2 removed. As shown in Fig. 5, the reaction time results from this alternative model were also much longer than the human results in both Level 2' and 3' conditions, especially when the number of road names was relatively large (twelve and eighteen road names respectively). In these cases without Assumption 2, the increased reaction time was caused by visually scanning through all the road names without using the shortcut. The MAPE increased to $43 \%$, and RMSE increased to $1.9 \mathrm{~s}$. This
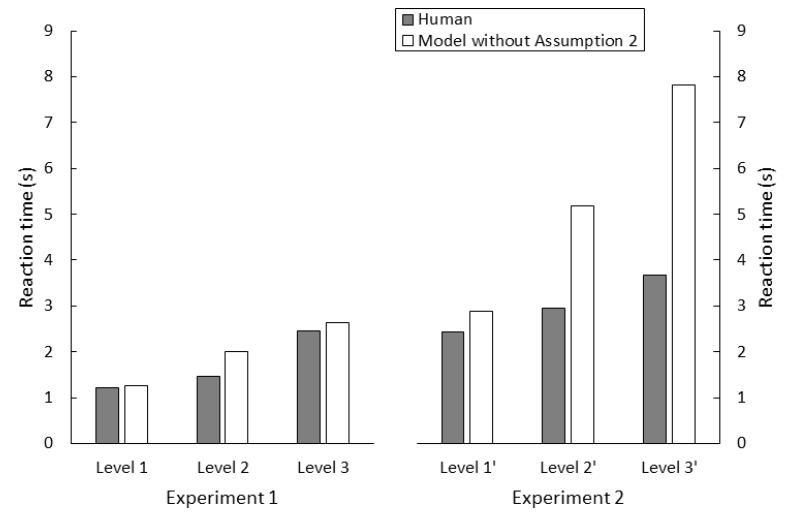

Fig. 5. Sign reading reaction time from different result showed that Assumption 2 is also necessary to accurately reflect human performance.

\section{Discussion}

An integrated cognitive architecture is both a unified theory of cognition and an engineering tool for the simulation of human performance. A model using an integrated cognitive architecture is a synthesized account for task environment, human knowledge, and human constraints. In this study, models were built in QN-ACTR to predict direction sign reading reaction time in both static and dynamic tests with different numbers of sign names. The models for both sign reading and driving task components closely followed previous models in the literature. In the sign reading model component, two assumptions about drivers' visual search strategy were proposed and tested. The results showed that both assumptions were necessary to produce reaction time results similar to the human data. The driving model component directly used the same one as in previous work [21, 23, 25]. All parameters were using their default values in the cognitive architecture, and we did not adjust any parameter to fit the human data. The model simulation results were similar to the human results, showing that the models had good predictive power. It also demonstrated a benefit of the cognitive architecture approach. The models, methods, and parameters established in completed work can be used in future modeling work that needs to simulate human performance in similar tasks. The capability and predictive power of the architecture grow as more studies accumulate more research outcomes in the integrated cognitive architecture.

A major value of computational cognitive architecture models is to quantitatively predict human performance. Although the qualitative trend of model results (i.e., reading time increases as information volume) is not very surprising, the quantitative prediction is overall accurate across all the conditions (RMSE $=0.3 \mathrm{~s}$, MAPE $=$ $12 \%)$. It is also intuitive to expect sign reading time to be longer in dual-task conditions than single-task conditions, but how much longer is the question to be answered by computational models. In this regard, Hick-Hyman law cannot predict the time increase in the dual-task condition, but the current modeling approach can. In future studies, we plan to continue testing models for more complex traffic signs that combine multiple categories of information [32]. It will also be interesting to examine the impact of other human factors such as driving experience [33] and concurrent mobile phone tasks [34] on sign reading performance. Previous models and the current models can be combined and tested under the same QN-ACTR framework.

The dual-task model in the current study was formed by combining the two single-task models following the multitask scheduling mechanisms in QN-ACTR including the filtering discipline. The initial motivation of QN-ACTR research is to combine QN's multitask scheduling mechanisms with ACT-R's production rule system [13], because the ACT-R cognitive architecture has focused on complex cognitive activities such as memory and learning mostly in single-task scenarios. The results from the current study demonstrated the value of integrating QN and ACT-R. Previous work using QN-ACTR has tested the filtering 
discipline in the simulation of a diagnostic decision and auditory concurrent task scenario [31] as well as a driving with auditory sentence comprehension dual-task scenario [25]. In the current study, we tested a new scenario, which was driving with visual sign reading dual-task scenario. The results showed again that QN-ACTR's multitask scheduling mechanisms are valid and effective.

Because the multitask scheduling mechanisms in QN-ACTR is domain independent, we expect that other driving circumstances can also be modelled using the same approach. In another study submitted under review [35], we applied the approach to model take-over transfer tasks where drivers must re-engage manual driving in an autonomous vehicle following a take-over request. The results showed that the models could simulate drivers' take-over reaction time in different conditions with or without a visual or auditory secondary task. Future studies are needed to model drivers' sign reading with other types of traffic signs. For signs with pictures or icons, the graphical components need to be manually encoded by researchers for the model to understand. Alternatively, image recognition tools can be used, but such tools are currently not part of the cognitive architecture and need to be developed in the future.

Previous entropy-based models were regression models that estimate reaction time based on only one factor, which is the amount of information. In contrast, the current models are cognitive simulation models that can take multiple factors into account, such as road condition, traffic condition, driver fatigue, and driver attention. These factors can be programmed in the simulation platform, and models can interact with different driving conditions under different assumptions about the mental capacity of the drivers. This is an advantage of the QN-ACTR models for sign reading reaction time prediction.

A limitation of the current models is that only one strategy was used. Different drivers may have different strategies, and even the same driver may use different strategies in different trials. The choice of strategies may be a reason explaining the remaining differences of reaction time between the model results and the human data. Since strategies are represented as production rules, a way to address mixed strategies is to program multiple models that use different sets of production rules. Previous work has used different production rules to represent different strategies used by novice and experienced drivers [22] as well as different strategies in a diagnostic decision task [31]. Future research can use a weighted average of results from models using different production rules to represent multiple strategies used by human operators. Although the two assumptions about drivers' visual search and response strategies seem to be reasonable and necessary for the modeling results, future studies are needed to collect empirical evidence using eye tracking measures to confirm both assumptions. The current study focused on computationally modeling direction sign reading reaction time rather than validating the assumptions.

Another limitation of the current study is that human error was not modeled. The human responses from the experiments were not all correct. However, we chose to omit the errors and focus on modeling reaction time because it is difficult to model errors without knowing the sources of errors. In a cognitive architecture, sources of errors include perceptual errors, memory errors, decision errors, and motor errors. Future studies focusing on modeling errors need to first analyze the sources of errors in the human data, which is not an easy task. After knowing the error sources or having assumptions about the sources, future work can build models and simulate erroneous performance together with reaction time performance.

\section{Conclusions}

QN-ACTR models were built to predict drivers' direction sign reading reaction time in both static and dynamic tests. Drivers' task-specific knowledge and skills were programmed as production rules. Two assumptions about drivers' strategies were proposed and tested. The models were built using existing modeling methods without adjusting any parameter to fit the human data. The models' prediction was similar to the human data and could capture the different reaction time in different task conditions with different numbers of road names on the direction signs. The results supported the modeling assumptions and demonstrated the models' predictive power. The models provide a useful tool for the prediction of driver performance and evaluation of direction sign design. The driving model from the current study can support future work that simulates driving performance in other tasks and traffic environments.

\section{Acknowledgments}

The research was supported by National Natural Science Foundation of China (51678460, U1664262); Open Project of Key Laboratory of Ministry of Public Security for Road Traffic Safety (2017ZDSYSKFKT02); Natural Science Foundation of Hubei Province, China (ZRMS2017001571); Wuhan Youth Science and Technology Plan (2017050304010268); Fundamental Research Funds for the Central Universities (2017-JL-003). This work was supported in part by Natural Sciences and Engineering Research Council of Canada Discovery Grant RGPIN-2015-04134 (to SC).

\section{References}

1 Guo, Z., Wei, Z., Wang, H.: 'The expressway traffic sign information volume threshold and AGS position based on driving behaviour'Transportation Research Procedia, 2016, 14, pp. 3801-3810.

2 Federal Highway Administration: 'Manual on uniform traffic control devices' (United States Department of Transportation, Washington, D.C., 2009)

3 Dutta, A., Fisher, D.L., Noyce, D.A.: 'Use of a driving simulator to evaluate and optimize factors affecting understandability of variable message signs'Transportation Research Part F: Traffic Psychology and Behaviour, 2004, 7, (4-5), pp. 209-227.

4 Du, Z.G., Pan, X.D., Guo, X.B.: 'Relationship between information quantity and visual cognition of traffic guide sign'Journal of Traffic and Transportation Engineering, 2008, 8, (1), pp. 118-122. 
5 Lyu, N., Xie, L., Wu, C., Fu, Q., Deng, C.: 'Driver's cognitive workload and driving performance under traffic sign information exposure in complex environments: A case study of the highways in China'International Journal of Environmental Research and Public Health, 2017, 14, (2), p. 203.

6 Zwahlen, H.T.: 'Traffic sign reading distances and times during night driving'Transportation research record, 1995, (1495), pp. 140-146.

7 Shannon, C.E.: 'A mathematical theory of communication' Bell system technical journal, 1948, 27, (3), pp. 379-423.

8 Fu, Q.: 'Study on the effect of driving information load to road traffic safety in highway ramp area'. Wuhan University of Technology, 2014

9 Hyman, R.: 'Stimulus information as a determinant of reaction time' Journal of Experimental Psychology, 1953, 45, (3), pp. 188-196.

10Wickens, C.D., Hollands, J.G., Banbury, S., Parasuraman, R.: 'Engineering psychology and human performance' (Pearson, 2013)

11 Anderson, J.R., Bothell, D., Byrne, M.D., Douglass, S., Lebiere, C., Qin, Y.: 'An integrated theory of the mind'Psychological Review, 2004, 111, (4), pp. 10361060 .

12 Laird, J.E., Newell, A., Rosenbloom, P.S.: 'Soar: An architecture for general intelligence' Artificial Intelligence, 1987, 33, (1), pp. 1-64.

13 Cao, S., Liu, Y.: 'Queueing network-adaptive control of thought rational (QN-ACTR): An integrated cognitive architecture for modelling complex cognitive and multi-task performance'International Journal of Human Factors Modelling and Simulation, 2013, 4, (1), pp. 6386.

14 Liu, Y., Feyen, R., Tsimhoni, O.: 'Queueing Network-model human processor (QN-MHP):A computational architecture for multitask performance in human-machine systems' $A C M$ Transactions on Computer-Human Interaction, 2006, 13, (1), pp. 37-70.

15 Liu, Y.: 'Queueing network modeling of elementary mental processes' Psychological Review, 1996, 103, (1), pp. 116-136.

16 Liu, Y.: 'Queueing network modeling of human performance of concurrent spatial and verbal tasks' IEEE Transactions on Systems, Man, and Cybernetics - Part A: Systems and Humans, 1997, 27, (2), pp. 195-207.

17 Feyen, R.G.: 'Modeling human performance using the queuing network-model human processor (QN-MHP)' (University of Michigan, 2002)
18 Salvucci, D.D., Taatgen, N.A.: 'Threaded cognition: An integrated theory of concurrent multitasking.' Psychological Review, 2008, 115, (1), pp. 101-130.

19 Cao, S., Liu, Y.: 'Queueing network-adaptive control of thought rational (QN-ACTR): An integrated cognitive architecture for modelling complex cognitive and multi-task performance'International Journal of Human Factors Modelling and Simulation, 2013, 4, (1), pp. 6386.

20 Cao, S., Liu, Y.: 'Queueing Network-ACTR modeling of concurrent tasks involving multiple controlled processes'Proceedings of the Human Factors and Ergonomics Society Annual Meeting, 2013, 57, (1), pp. 768-772.

21 Salvucci, D.D.: 'Modeling driver behavior in a cognitive architecture'Human Factors, 2006, 48, (2), pp. 362-380.

22 Cao, S., Qin, Y., Jin, X., Zhao, L., Shen, M.: 'Effect of driving experience on collision avoidance braking: An experimental investigation and computational modelling'Behaviour \& Information Technology, 2014, 33, (9), pp. 929-940.

23 Cao, S., Qin, Y., Zhao, L., Shen, M.: 'Modeling the development of vehicle lateral control skills in a cognitive architecture'Transportation Research Part F: Traffic Psychology and Behaviour, 2015, 32, pp. 1-10.

24 Salvucci, D.D., Beltowska, J.: 'Effects of memory rehearsal on driver performance: Experiment and theoretical account'Human Factors, 2008, 50, (5), pp. 834-844.

25 Cao, S., Liu, Y.: 'Modeling driving and sentence comprehension dual-task performance in Queueing Network-ACTR' Proceedings of the Human Factors and Ergonomics Society Annual Meeting, 2014, 58, (1), pp. 808-811.

26 Wickens, C.D.: 'Multiple resources and mental workload'Human Factors, 2008, 50, (3), pp. 449-455.

27 Deng, C., Wu, C., Cao, S., Lyu, N.: 'Modeling the effect of limited sight distance through fog on car-following performance using QN-ACTR cognitive architecture' Transportation Research Part F Traffic Psychology and Behaviour, 2018, p. In press.

28 Standardization Administration of the People's Republic of China: 'Road Traffic Signs and Markings Part 2: Road Traffic Signs, GB 5768.2-2009’ (2009)

29 Cao, S., Ho, A., He, J.: 'Modeling and predicting mobile phone touchscreen transcription typing using an integrated cognitive architecture'International Journal of Human-Computer Interaction, 2018, 34, (6), pp. 544556. 
30 Salvucci, D.D., Gray, R.: 'A two-point visual control model of steering'Perception, 2004, 33, (10), p. 1233.

31 Cao, S., Liu, Y.: 'Queueing Network-ACTR modeling of concurrent tasks involving multiple controlled processes' Proceedings of the Human Factors and Ergonomics Society Annual Meeting, 2013, 57, (1), pp. 768-772.

32 Liu, X., Wang, W., Jiang, M., Wei, Z.: 'Investigation of information quantity threshold on combined traffic signs' Journal of Traffic and Transportation Engineering, 2016, 16, (1), pp. 141-148.

33 Cao, S., Qin, Y., Zhao, L., Shen, M.: 'Modeling the development of vehicle lateral control skills in a cognitive architecture' Transportation Research Part $F$ Traffic Psychology and Behaviour, 2015, 32, pp. 1-10.

34 Cao, S., Ho, A., He, J.: 'Modeling and predicting mobile phone touchscreen transcription typing using an integrated cognitive architecture'International Journal of Human-Computer Interaction, 2017, pp. 1-13.

35 Deng, Cao, S., Wu, C.: 'Modeling Driver Take-over Reaction Time Using an Integrated Cognitive Architecture'TRR, no date. 